九州大学学術情報リポジトリ

Kyushu University Institutional Repository

\title{
A virtual modeling system for intuitive 3D shape conceptualization
}

Nishino, Hiroaki

0ita University

高木，英行

Department of Art and Information Design, Kyushu Institute of Design

SAGA, Sato

Muroran Institute of Technology

Utsumiya, Kouichi

0ita University

http://hdl. hand le. net/2324/4486303

出版情報: 2002 IEEE International Conference on Systems, Man, and Cybernetics. 4, pp.541-546, 2002-10-06. IEEE

バージョン :

権利関係 : ๑ 2001 IEEE. Personal use of this material is permitted. Permission from IEEE must be obtained for all other uses, in any current or future media, including reprinting/republishing this material for advertising or promotional purposes, creating new collective works, for resale or redistribution to servers or lists, or reuse of any copyrighted component of this work in other works. 


\title{
A Virtual Modeling System for Intuitive 3D Shape Conceptualization
}

\author{
${ }^{* 1}$ Hiroaki NISHINO $\quad{ }^{2}$ Hideyuki TAKAGI $\quad{ }^{3}$ Sato SAGA $\quad{ }^{4}$ Kouichi UTSUMIYA
}

\author{
${ }^{1,4}$ Oita University, 700 Dannoharu, Oita 870-1192, Japan \\ ${ }^{2}$ Kyushu Institute of Design, 4-9-1 Shiobaru Minami-ku, Fukuoka 815-8540, Japan \\ ${ }^{3}$ Muroran Institute of Technology, 27-1 Mizumoto-cho, Muroran 050-8585, Japan \\ Email: ${ }^{1}$ hn@csis.oita-u.ac.jp, ${ }^{2}$ takagi@kyushu-id.ac.jp, ${ }^{3}$ saga@csse.muroran-it.ac.jp, ${ }^{4}$ utsumiya@csis.oita-u.ac.jp
}

\begin{abstract}
We propose an approach to build a 3D modeling environment for aiding new shape conceptualization. The proposed system consists of the following four functional components: (a) a freehand sketch interface to allow the user to easily bring his/her rough design idea into a 2D geometric model, (b) a shape approximation module (SAM) to automatically convert the sketched 2D image into a 3D deformable model, (c) a 3D shape exploration tool to generate variety of 3D models by evolving the converted 3D model, and (d) a parametric 3D modeler to sophisticate the evolved shapes. The freehand sketch interface employs a fuzzy inference technique to flexibly identify a user's handwritten image as one of seven geometric primitives commonly used in many CAD tools. After the freehand sketch is automatically converted to a 3D model by the SAM, the model is evolved by using a technique called Interactive Evolutionary Computation (IEC). IEC automatically generates and deforms the model according to the user's rating and allows even beginners to easily produce interesting shapes. Advanced users and experts are encouraged to use parametric modeler to finish the model with elaboration.
\end{abstract}

Index terms-3D modeling, freehand sketch interface, interactive evolutionary computation, computer graphics.

\section{INTRODUCTION}

We propose a virtual modeling system to make the 3D model design an intuitive task for everyone. Although a large variety of sophisticated CAD software becomes widely available on the personal computer, the creation of aesthetically pleasing 3D shape remains a hardest problem.

One of the most serious problem is that these CAD tools require a certain knowledge and expertise on geometry and computer graphics (CG) theories to effectively manage hundreds of esoteric commands. Therefore, the user need to compose a coordinate system in his/her mind to geometrically represent a 3D shape he/she wants to produce, and accurately enter all geometric parameters of the shape to make it visible on the computer display. Because this traditional way of 3D modeling forces the user to think and act like computers as shown on the left side of figure 1, he/she cannot concentrate on getting an idea from imagination.

We introduce a freehand sketch and an idea evocation interfaces to assist the user in easy and intuitive 3D modeling as shown on the right side of figure 1 . The freehand sketch interface (FHSI) allows the user to easily outline his/her idea just like a regular painting. Next, the system converts the handwritten image into a set of geometric primitives like

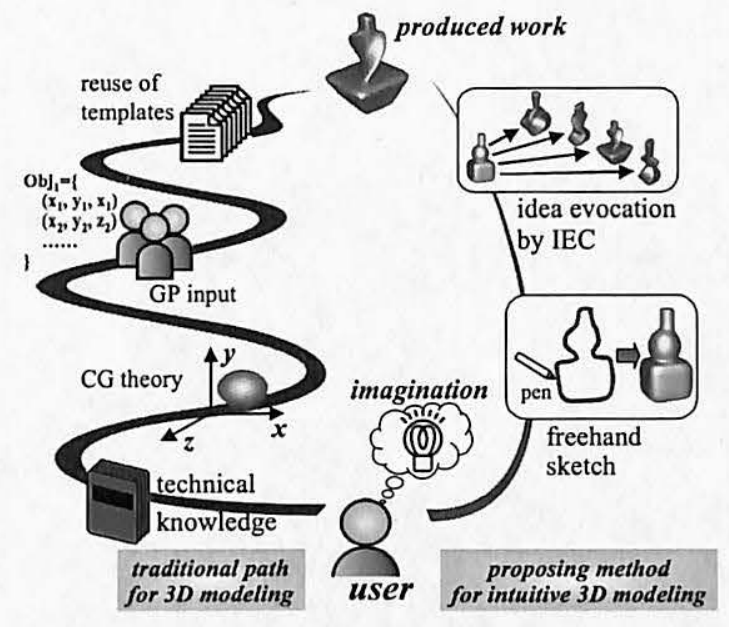

CG: computer graphics, GP: geometric parameter
IEC: interactive evolutionary computation

Figure 1: Proposed system concept.

ellipses and free-form curves by using a fuzzy inference technique. Then, the converted primitives are used to extract a deformable model represented by a mathematical function called superquadrics. Finally, the idea evocation interface transforms the extracted model to various patterns by using a technique called Interactive Evolutionary Computation (IEC). Therefore, the fuzzy-based FHSI and the IEC-based 3D shape explorer form an integrated core technology of the proposed modeling system. A parametric 3D modeler is also provided for advanced users and professionals to manually sophisticate the evolved models. The resulting system effectively aids the user to intuitively creating tangible shapes from imagination.

\section{RELATED RESEARCH WORK}

There are several prior research projects on the FHSI. Zeleznik [1] and Eggli [2] proposed the automatic conversion methods from a $2 \mathrm{D}$ planar sketch to a corresponding $3 \mathrm{D}$ model. Their methods, however, only deal with 2D sketches painted by straight lines. Igarashi's Teddy system [3] provides a nicer conversion function to generate more variety of 3D shapes from curvilinear drawings. The user, however, needs to be familiar with the Teddy's conversion algorithm to generate a 3D model matching with his/her imagination. 


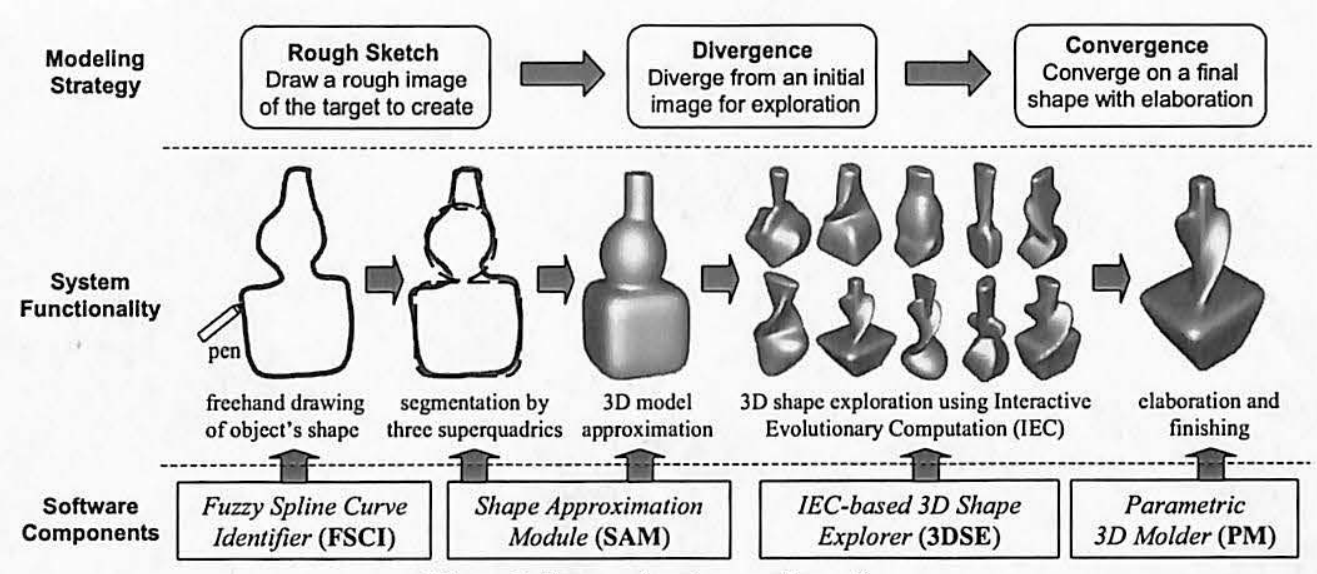

Figure 2: Proposed system configuration.

Additionally, some operations indispensable for making complex shapes such as deformation and blending are not available in the Teddy system.

Many IEC-based CG modeling systems have also been proposed as solutions to realize easy-to-use $2 \mathrm{D}$ and $3 \mathrm{D}$ modeling environments [4]. Dawkins has demonstrated the power of computer-simulated evolution in his Boimorphs software, a 2D line drawing system of complex structures [5]. Sims [6] and Unemi [7] individually developed their own graphics art applications based on a concept, the so-called simulated breeding, to generate aesthetic graphics images based on artificial selection rather than natural selection. Other artistic applications are: line painting systems targeted at generating insects, plants, and human face drawings: interactive EC-based CG rendering systems for artificial life art, animals, and plants: 3D CG lighting design support system: industrial designs. See the reference [4] for further detail survey. The target shapes of these systems are highly abstract or application-specific images to be easily associated with the simulated evolution.

The FHSI is clearly beneficial to externalize the user's imagination into a concrete 3D model. It is, however, difficult to produce highly abstract or unsketchable shapes. On the contrary, IEC is best suitable for model production from vague images, but it is not sufficient for elaboration of the target shapes.

We show an approach to integrate the FHSI and the IECbased modeling in a uniform fashion. The proposed system is realized based on the $3 \mathrm{D}$ modeler we have continuously been implemented and improved for last few years [8][9]. Because the system provides simple yet powerful $3 \mathrm{D}$ modeling operations, it is able to support all sorts of people from professional designers to novices and from adults to children.

\section{IMPLEMENTATION METHOD}

Figure 2 shows the proposed system configuration with an example of "vase" modeling. The system provides the threephase modeling strategy consisting of the rough sketch, the divergence, and the convergence phases as indicated in the figure. This modeling strategy is supported by four software components such as the fuzzy spline curve identifier (FSCI), the shape approximation module (SAM), the IEC-based $3 D$ shape explorer (3DSE), and the parametric $3 D$ modeler

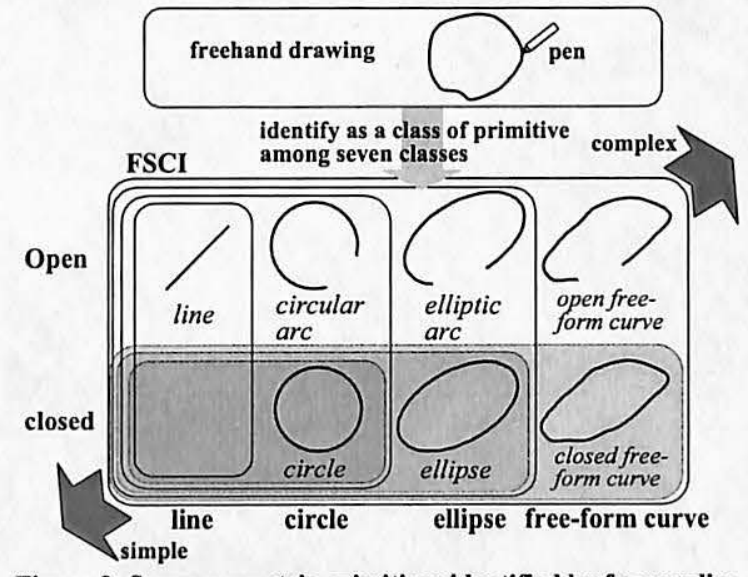

Figure 3: Seven geometric primitives identified by fuzzy spline curve identifier (FSCI).

(PM). The following subsections elaborate these components' functions and inside implementation.

\section{A. Freehand Sketch Interface by FSCI}

The FHSI assists the user to intuitively bring his/her unshaped design idea into a handwritten shape in the rough sketch phase. In this phase, a freehand drawing is identified and converted to a 2D geometric primitive by using a technique, the so-called fuzzy spline curve identifier (FSCI) [10][11]. The FSCI realizes a freehand curve identification technique to be widely available as a general-purpose shape input module for any $3 \mathrm{D}$ modeling engines. It classifies a freehand drawing into one of seven fundamental primitives usable in many CAD systems such as a line, a circle, a circular arc, an ellipse, an elliptic arc, a closed free-form curve, and an open free-form curve as shown in figure 3 . There is an inclusive relation among these primitives as shown in the figure. An upper right primitive is a superset of all other primitives in a quadrant.

Because it is difficult to flexibly classify these similar geometries, the traditional FHSI systems tend to only identify a single class primitive such as the line [1][2] or the freeform curve [3]. The FSCI is able to robustly classify a handwritten curve into a specific primitive among the seven alternatives by observing the user's drawing behavior. If he/she quickly paint a coarse curve, it is more likely to be 


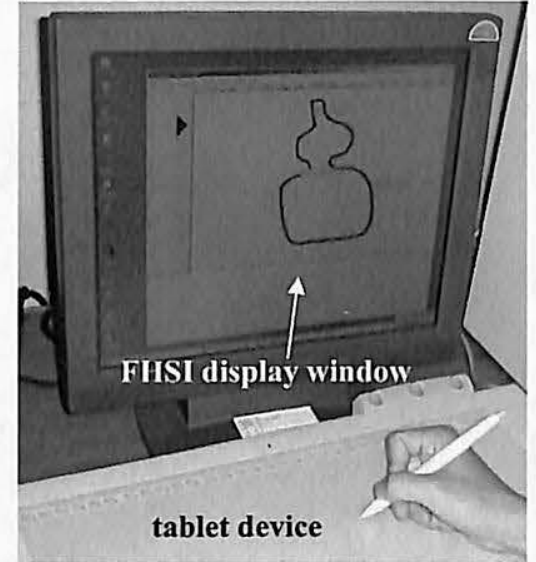

Figure 4: Freehand Sketch Interface (FHSI) with tablet device.

determined as a simpler primitive such as a line, a circle, or an ellipse. On the other hand, a carefully drawn exquisite curve tends to be estimated as a complex free-form curve. Therefore, the FSCI enables the user to intentionally control the result with the drawing speed and the fineness of his/her hand drawing operations.

Figure 4 shows a typical FHSI setup with a tablet device used for easy freehand drawing operations.

\section{B. Shape Approximation and $2 D$ to $3 D$ Conversion by SAM}

A freehand drawing identified as a geometric primitive by the FSCI is used as a reference model to generate various shapes in the divergence phase. A 3D deformable model approximating the $2 \mathrm{D}$ handwritten shape is required to start the geometrical exploration. The shape approximation module (SAM) carries out the approximation and the 2D to 3D conversion.

Figure 5 illustrates how the SAM works in the case of the vase example in figure 2 . The handwritten vase is identified as a closed free-form curve by the FSCI. The SAM divides the curve into a set of 2D deformable parts by using the superquadrics expansion method [12][13]. Each part is represented by a mathematical function called superquadrics that is formulated by the following implicit equation:

$$
\left(x / r_{x}\right)^{2 / e}+\left(y / r_{y}\right)^{2 / e}=1 \text {. }
$$

where $r_{x}$ and $r_{y}$ are the scale parameters to control the size of the primitive, $e$ is the squareness parameter to control the shape as shown in figure 6(a).

At the beginning of the approximation procedure, a superquadrics primitive is set at the barycenter or a userspecified point inside the curve as shown in figure 5(b). Then, the primitive is expanded until it approximates a certain part of the curve as shown in figure 5(c). The primitive shape and its position are dynamically adjusted to match with as larger portion of the curve as possible. A nonlinear least square method is used to approximate a part of the curve with a superquadrics primitive [12]. Then this process is iterated for the remaining portion until the curve is completely segmented and approximated by the primitives as

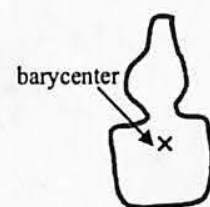

(a) a closed curve identified by FSCI

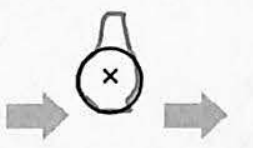

(d) segment the medium part

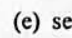

e) segment
top part

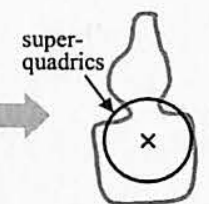

(b) expand a 2D superinside a curve quadrics primitive

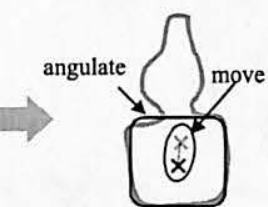

(c) segment the bottom part by an angulated superquadrics
Figure 5: Shape approximation by using superquadrics.
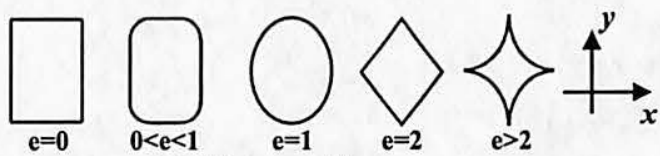

(a) 2D superellipse
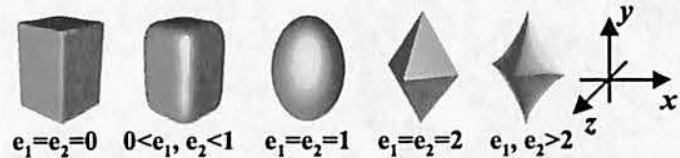

(b) $3 \mathrm{D}$ superellipsoid

Figure 6: 2D and 3D superquadrics.

illustrated in figure 5(d) and 5(e). Figure 5(f) shows a result of the approximation by the SAM.

Next, the SAM converts the acquired 2D model to a corresponding 3D model. A 2D superquadrics primitive used for the approximation process can easily be generalized to a 3D superquadrics primitive as follows:

$$
\left\{\left(x / r_{x}\right)^{2 / e z}+\left(y / r_{y}\right)^{2 / e z}\right\}^{e 2 / e l}+\left(z / r_{z}\right)^{2 / e I}=1 .
$$

where $r_{x}, r_{y}$, and $r_{z}$ are the scale parameters, $e_{1}$ and $e_{2}$ are the squareness parameters. Figure $6(\mathrm{~b})$ shows the expressible shapes of the 3D superquadrics in contrast with their 2D counterparts. A 2D primitive can be transformed to its equivalent $3 \mathrm{D}$ shape by substituting the $2 \mathrm{D}$ squareness value $\boldsymbol{e}$ for the corresponding 3D parameters $e_{1}$ and $e_{2}$. Additionally, the depth value $r_{z}$ is set to a countervalue of the width $r_{x}$. Finally, the resultant 3D model as shown in figure $5(\mathrm{~g})$ is obtained.

\section{Shape Exploration by 3DSE}

The generated 3D model is used as an initial model to evolve the shape in the divergence phase. The IEC-based 3D shape explorer (3DSE) provides the user with a mean to intuitively create novel geometries. It takes the initial model as an input to trigger the shape exploration and automatically derives various shapes by simulating natural evolution [8][9]. The 3DSE simultaneously displays up to twenty evolved models as shown in figure 7 . The user can easily find his/her 


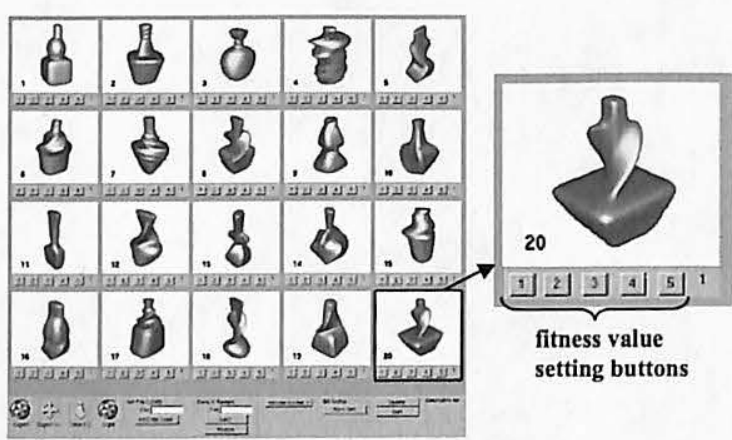

Figure 7: IEC-based 3D Shape Explorer.

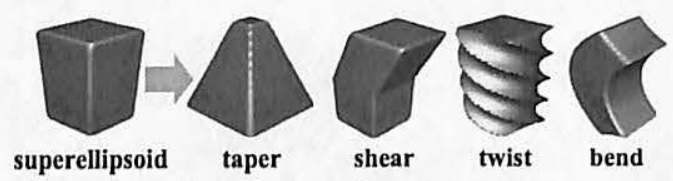

(a) Enhanced primitive deformations
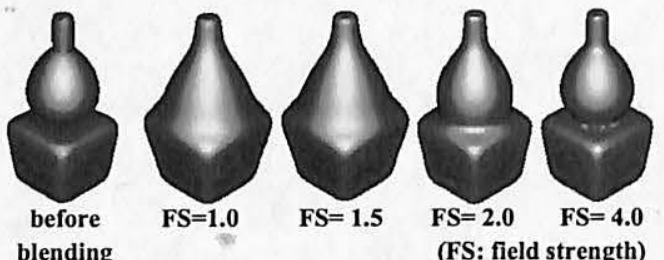

(b) Primitive blending by implicit surface method

Figure 8: Primitive deformation and blending.

preferred shapes among them, getting inspirations about how to evolve the shapes to get more appealing ones, and subjectively controlling the evolution process by rating the preferred shapes highly. Then the 3DSE re-execute the evolution process based on the given rates to generate better shapes. This "rating-evolution" cycle is iterated until the exploration converges on a satisfactory result.

1) Manipulated Geometric Parameters

Table 1 shows all geometric parameters to define a superquadrics primitive in the 3DSE. There are a dozen parameters in addition to the scale and the squareness parameters as described in the previous subsection. A set of parameters to control the primitive position and the orientation in a global coordinate system, and some finer deformation parameters as shown in figure $8(a)$ are incorporated into the $3 \mathrm{D}$ superquadrics representation to generate full of diversity shapes [14]. While all of these parameters can be manipulated in the shape exploration process, the user can explicitly select specific parameters to apply for the new shape creation.

Multiple primitives are blended to make the resultant shape a smooth and natural one as shown in figure 8(b). A modeling technique called the implicit surface representation is used to realize the blending operation [14]. An additional parameter called the field strength controls the blending effect on boundaries between primitives as shown in the figure.

2) 3D Model Coding for Evolutionary Computation

We adopt genetic algorithm (GA) as one of the evolutionary computation technologies to evolve 3D models in the 3DSE [8][9]. The initial 3D model is encoded as a chromosome for

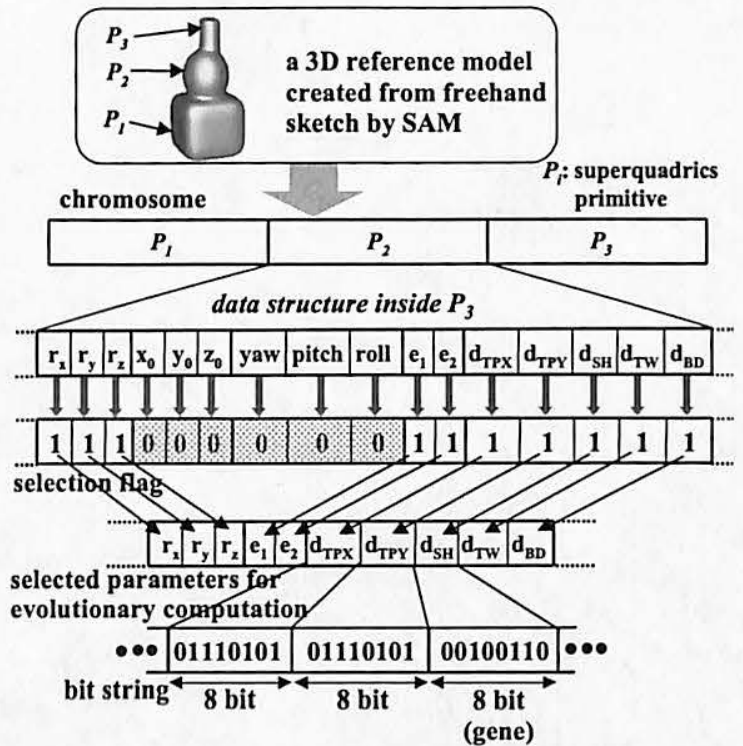

Figure 9: GA coding of a 3D model.

the simulated evolution as illustrated in figure 9. The selection flag allows the user to explicitly specify a subset of parameters modified by GA operations, and moderately control the shape exploration process toward his/her expectations. For example, the GA coding in figure 9 excludes the position and the orientation of the model by the selection flag to keep these values unchanged.

Each selected parameter listed in table 1 is encoded as an 8bit string called gene. The following equation defines a relation between the parameter and the corresponding gene:

$$
P=P_{\min }+\frac{P_{\max }-P_{\min }}{255} \times G .
$$

where $\boldsymbol{P}$ is a parameter value; $\boldsymbol{G}$ is an encoded gene of $P$; $\boldsymbol{P}_{\max }$ and $\boldsymbol{P}_{\min }$ are the parameter's maximum and minimum values.

Table 1: 3D superquadrics parameters to be handled by IEC.

\begin{tabular}{|c|c|c|}
\hline \multicolumn{2}{|c|}{ Parameter } & Roles and Functions \\
\hline \multicolumn{2}{|l|}{$r_{x}, r_{y}, r_{z}$} & scale \\
\hline \multicolumn{2}{|l|}{$e_{1}, e_{2}$} & squareness \\
\hline \multicolumn{2}{|l|}{$x_{0}, y_{0}, z_{0}$} & position in a global coordinate \\
\hline \multicolumn{2}{|c|}{ yaw, pitch, roll } & orientation \\
\hline \multirow{4}{*}{$\begin{array}{l}\text { enhanced } \\
\text { deformation }\end{array}$} & $d_{t p x}, d_{t p p}$ & tapering \\
\hline & $\boldsymbol{d}_{\text {sh }}$ & shearing \\
\hline & $d_{t w}$ & twisting \\
\hline & $d_{b d}$ & bending \\
\hline
\end{tabular}

\section{3) 3D Model Evolution Procedure}

First of all, the 3DSE creates an initial generation, the fixed number of encoded 3D models up to twenty, from which the GA-based evolutions start. It is made by randomizing the initial model's selected parameters until the user finds some shapes containing appropriate geometric features to emphasize in the following evolutions.

Then, the 3DSE starts the interactive GA process to evolve the initial generation. In this process, the user rates the fitness 


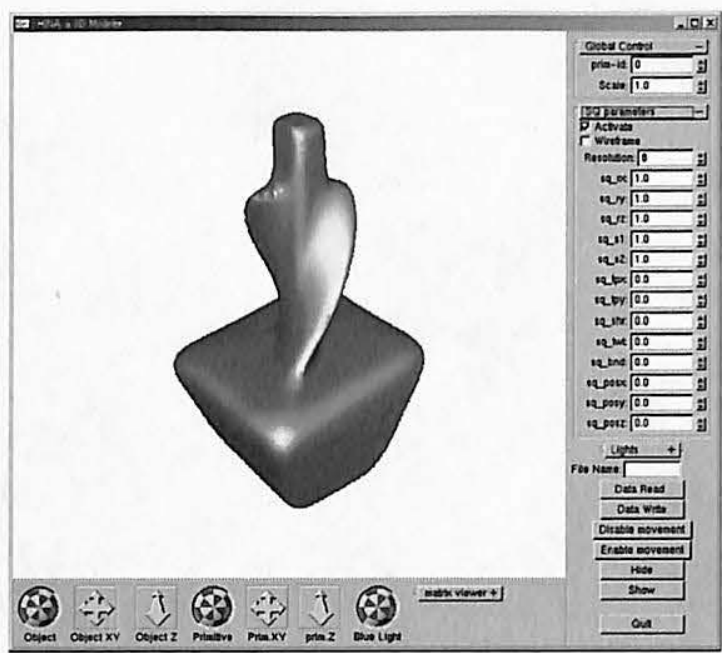

Figure 10: Parametric 3D modeler.

value for each evolved model according to his/her subjective preference. Then, a new generation of models (offsprings) is yielded from the current set of rated models (parents) by applying GA operations such as the crossover and the mutation. Highly rated models have high probability to survive, being selected as parents to generate new offsprings. Top $5 \%$ of such highly rated individuals survive in the new generation if the crossover rate is $95 \%$. This "evaluation and reproduction" process is iterated until an acceptable model shape is produced.

Some conditional variables required for the GA operations such as the selection flag, $\boldsymbol{P}_{\max }$ and $\boldsymbol{P}_{\min }$ in equation 3 for each selected parameter, and GA control variables like the crossover and the mutation rates need to be set by the user. Because the GA-based shape evolutions depend on the user's evaluation, the number of models in a generation is usually limited to not more than twenty to minimize the user's fatigue.

\section{4) Manual Sophistication by $P M$}

The 3DSE effectively evokes the user's imagination by presenting the diversified shapes via the interactive GA session. The user can easily find unexpected 3D shape variations and gradually converges with a specific one by highly rating it and its neighbors. The level of convergence achievable by the 3DSE, however, sometimes is not enough for advanced users who seek exquisite 3D representations. Therefore, the parametric 3D modeler (PM) is provided for such users to finish the whole modeling process by manually elaborating the best shape found in the IEC-based shape exploration. Figure 10 shows the look and feel of the PM. It provides a set of buttons and slider bars to directly modify the parameter values of an evolved 3D model [8]. Changing a specific parameter value via a GUI gismo instantly deform the corresponding primitive geometry in the model. The users who understand the meanings of all parameters can elaborate the model shape by subtly adjusting some parameter values.

\section{ANOTHER MODELING EXAMPLE}

In the previous section, we elaborate the proposed method by reviewing each modeling process through the vase example.
In this section, we present another modeling example to show the ability and the effectiveness of the proposed method.

Figure 11 shows another example targeted at designing stuffed animals. A handwritten sketch as shown in figure 11(a) is approximated by eight $2 \mathrm{D}$ primitives and then converted to a 3D reference model as indicated in figure $11(\mathrm{~b})$ and 11 (c), respectively. Figure 11(d) shows the blended reference model. Several models generated from the reference model by using the IEC-based 3DSE are exhibited in figure 11(e).

The 3DSE executes the shape evolution under the following conditions:

- eight superquadrics primitives are used,

- the scale $\left(r_{x}, r_{y}, r_{z}\right.$ as shown in table 1$)$, the squareness $\left(e_{l}\right.$, $\left.e_{2}\right)$, and the five enhanced deformations $\left(d_{t p x}, d_{t p y}, d_{s h}, d_{t w}\right.$ $d_{b d}$ ) parameters are selected for adjustments by the GA operations (the position and the orientation are kept unchanged to prevent the generated models from being evolved to absolutely different shapes from the reference model),

- the number of models evolved in a generation (population) is 20 ,

- the crossover rate is $95 \%$,

- the mutation rate is $1 \%$, and

- the fitness value is a rate on a scale of one to five (the worst to the best correspond to one to five).

While 19 offsprings ( $95 \%$ ) out of 20 models are generated by the crossover operation in every generation, the only one individual $(5 \%)$ is treated as a highly rated elite parent to survive in the new generation.

The whole modeling tasks shown in figure 11 are performed by a single subject who has some $3 \mathrm{D}$ modeling experiences. The whole modeling time including the creation of a dozen evolved shapes (only a part of the models are shown in figure $11(\mathrm{e})$ ) is less than an hour. Because most models explored by the 3DSE finish up within ten iterations of the "ratingevolution" cycle, each model is yielded in a few minutes in the evolution process.

A fascinating characteristic among several points indicated by the subject is the system's capability to generate varied and inartificial shapes that are difficult to create by traditional CAD tools. Many high-end commercial tools require the users to master hundreds of esoteric commands before they start their work. Additionally, prominent skills and senses are mandatory to make the complex shapes as those illustrated in figure 11(e) by using such commercial tools. On the other hand, no special knowledge and techniques except for the simple sketching and the nonlogical decision-making are required to use our modeling system. While this example does not show the final elaboration by the parametric 3D modeler (PM), it should be possible to make the produced models more appealing ones by the PM.

More comprehensive modeling experiments directing to the use by different purposes and subjects with various skill levels need to be conducted. Because the system efficiency highly depends on the user's performance to intuitively sketch and perceptually evaluate their preferred shapes, studies on more elegant and fun-to-use GUI are very important future work as well. 


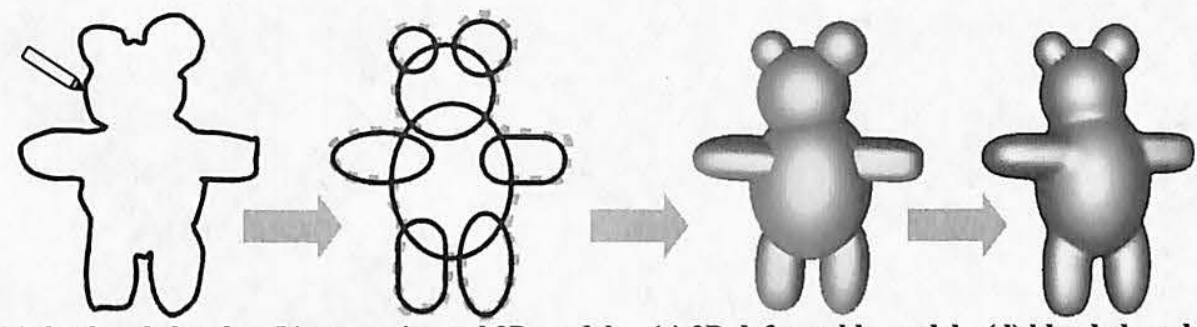

(a) freehand sketch

(b) approximated 2D model

(c) 3D deformable model

(d) blended model

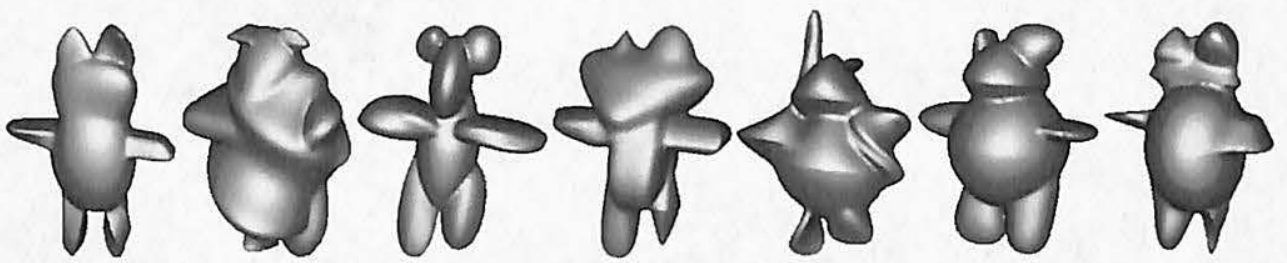

(e) derived models by IEC-based 3D shape explorer

Figure 11: A modeling example of stuffed animals.

\section{CONCLUSION}

We described the purpose, the characteristics, the implementation method, and the experimental results of the proposed 3D modeling system. The fuzzy-based freehand sketch interface allows the user to easily rough out his/her ideas and imagination in a normal 2D painting environment. Then, the 2D sketch is automatically converted to a deformable 3D model and the model is further evolved to breed new shapes through the technique called interactive evolutionary computation.

The results of the preliminary experiments suggest the proposed modeling method is quite useful for even beginners to create unprecedented shapes. Learning the validity in case of professional modeling is a future task. Currently, the freehand sketch interface and the IEC-based 3D shape explorer are implemented as independent tools. Although the user can simultaneously run both tools and dynamically switch them back and forth during the modeling task, integrating these tools into a single unified modeler is another future work. We also have an enhancement plan to make the system be accessible via the Internet, applying to the problems such as remote training and education, collaborative design, and 3D data sharing and distribution.

\section{ACKNOWLEDGEMENT}

This work is partly supported by the Grant-in-Aid for Scientific Research under Grant No.14580384 and No.14580451 by Ministry of Education, Culture, Sports, Science, and Technology.

\section{REFERENCE}

[1] Zeleznik, R., Herndon, K., and Hughes, J.: SKETCH: An Interface for Sketching 3D Scenes, Proc. of ACM SIGGRAPH'96, pp.163-170, 1996.

[2] Eggli, L., Hsu, C., Elber, G., and Bruderlin, B.: Inferring 3D Models from Freehand Sketches and Constrains, Computer-Aided Design, Vol.29, No.2, pp.101-112, 1997.
[3] Igarashi, T., Matsuoka, S., and Tanaka, H.: Teddy: A Sketching Interface for 3D Freeform Design, Proc. of ACM SIGGRAPH'99, pp.409-416, 1999.

[4] Takagi, H.: Interactive Evolutionary Computation: Fusion of the Capacities of EC Optimization and Human Evaluation, Proceedings of the IEEE, Vo.89, No.9, pp.1275-1296, 2001.

[5] Dawkins, R.: The Blind Watchmaker, Longman, Essex, 1986.

[6] Sims, K.: Artificial Evolution for Computer Graphics, Proc. of ACM SIGGRAPH'91, pp.319-328, 1991.

[7] Unemi, T.: A Design of Multi-Field User Interface for Simulated Breeding, Proc. 3rd Asian Fussy System Symposium, pp.489-494, 1998.

[8] Nishino, H., Takagi, H., Cho, S.-B., and Utsumiya, K.: A 3D Modeling System for Creative Design, Proc. of IEEE ICOIN-15, pp. 479-486, 2001

[9] Nishino, H., Takagi, H., and Utsumiya, K. Implementation and Evaluation of an IEC-Based 3D Modeling System, Proc. of IEEE SMC'01, pp. $1047-$ $1052,2001$.

[10] Saga, S. and Makino, H.: Fuzzy Spline Interpolation and Its Application to On-Line Free-hand Curve Identification, Proc. 2nd IEEE Int. Conf. On Fuzzy Systems, pp.1183-1190, 1993.

[11] Saga, S.: A Freehand Interface for Computer Aided Drawing Systems Based on the Fuzzy Spline Curve Identifier, Proc. of IEEE SMC'95, pp. 2754-2759, 1995.

[12] Horikoshi, T., Suenaga, Y., and Kaneko, K.: 3D Shape Description Using Superquadrics Expansion Method and Spherical Harmonics, Trans. of the IEICE, Vol.J78-D-II, No.1, pp.50-60, 1995. (in Japanese)

[13] Shinozaki, J., Hukunaga, S., Nishino, H., and Utsumiya, K.: A 3D Modeler Using Genetic Algorithm, Proc. IPSJ Kyushu Chapter Hi-no-kuni Information Symposium, pp.143-148, 2002.

[14] Nishino, H., Utsumiya, K., Sakamoto, A., Yoshida, K., and Korida, K.: A Method for Sharing Interactive Deformations in Collaborative 3D Modeling, Proc. of ACM VRST'99, pp.116-123, 1999. 